\title{
A walking programme and a supervised exercise class versus usual physiotherapy for chronic low back pain: a single-blinded randomised controlled trial. (The Supervised Walking In comparison to Fitness Training for Back Pain (SWIFT) Trial) Deirdre A Hurley*1, Grainne O'Donoghue ${ }^{1}$, Mark A Tully², Jennifer Klaber Moffett ${ }^{3}$, Willem van Mechelen ${ }^{4}$, Leslie Daly ${ }^{5}$, Colin AG Boreham ${ }^{6}$ and Suzanne M McDonough ${ }^{7}$
}

Address: ${ }^{1}$ School of Physiotherapy \& Performance Science, University College Dublin, Dublin 4, Ireland, ${ }^{2}$ Centre of Excellence for Public Health (Northern Ireland), Queens University Belfast, Mulhouse, Royal Victoria Hospital, Grosvenor Road, Belfast, BT12 6BJ, Northern Ireland, ${ }^{3}$ Institute of Rehabilitation, University of Hull, 215 Analaby Road, Hull, HU3 2PG, UK, ${ }^{4}$ EMGO Institute and Department of Public Health and

Occupational Health, VU University Medical Center, Amsterdam, The Netherlands, ${ }^{5}$ School of Public Health and Population Science, University College Dublin, Dublin 4, Ireland, ${ }^{6}$ Institute for Sport and Health, Phillips Building, University College Dublin, Dublin 4, Ireland and ${ }^{7}$ Health and Rehabilitation Sciences Research Institute, University of Ulster, Jordanstown Campus, Antrim, BT37 0QB, Northern Ireland

Email: Deirdre A Hurley* - deirdre.hurleyosing@ucd.ie; Grainne O'Donoghue - grainne.odonoghue@ucd.ie; Mark A Tully - m.tully@qub.ac.uk; Jennifer Klaber Moffett - J.K.Moffett@hull.ac.uk; Willem van Mechelen - w.vanmechelen@vumc.nl; Leslie Daly - leslie.daly@ucd.ie;

Colin AG Boreham - colin.boreham@ucd.ie; Suzanne M McDonough - s.mcdonough@ulster.ac.uk

* Corresponding author

This article is available from: http://www.biomedcentral.com/I47I-2474//0/79

(c) 2009 Hurley et al; licensee BioMed Central Ltd.

This is an Open Access article distributed under the terms of the Creative Commons Attribution License (http://creativecommons.org/licenses/by/2.0), which permits unrestricted use, distribution, and reproduction in any medium, provided the original work is properly cited.

\begin{abstract}
Background: Chronic low back pain (CLBP) is a persistent disabling condition with rising significant healthcare, social and economic costs. Current research supports the use of exercisebased treatment approaches that encourage people with CLBP to assume a physically active role in their recovery. While international clinical guidelines and systematic reviews for CLBP support supervised group exercise as an attractive first-line option for treating large numbers of CLBP patients at low cost, barriers to their delivery include space and time restrictions in healthcare settings and poor patient attendance. The European Clinical Guidelines have identified the need for research in the use of brief/minimal contact self-activation interventions that encourage participation in physical activity for CLBP. Walking may be an ideally suited form of individualized exercise prescription as it is easy to do, requires no special skills or facilities, and is achievable by virtually all ages with little risk of injury, but its effectiveness for LBP is unproven.
\end{abstract}

Methods and design: This study will be an assessor-blinded randomized controlled trial that will investigate the difference in clinical effectiveness and costs of an individualized walking programme and a supervised general exercise programme compared to usual physiotherapy, which will act as the control group, in people with chronic low back pain. A sample of 246 patients will be recruited in Dublin, Ireland through acute general hospital outpatient physiotherapy departments that provide treatment for people with CLBP. Patients will be randomly allocated to one of the three groups in a concealed manner. The main outcomes will be functional disability, pain, quality of life, fear avoidance, back beliefs, physical activity, satisfaction and costs, which will be evaluated at 
baseline, and 3, 6 and 12 months [follow-up by pre-paid postage]. Qualitative telephone interviews and focus groups will be embedded in the research design to obtain feedback about participants' experiences of the interventions and trial participation, and to inform interpretation of the quantitative data. Planned analysis will be by intention to treat (quantitative data) and thematic analysis (qualitative data)

Discussion: The trial will evaluate the effectiveness of a walking programme and a supervised general exercise programme compared to usual physiotherapy in people with CLBP.

\section{Trial registration: Current controlled trial ISRCTNI7592092}

\section{Background}

Chronic low back pain (CLBP) is a persistent disabling condition with rising significant healthcare, social and economic costs [1,2]. Current research and both European and American Clinical Guidelines supports the use of exercise-based treatment approaches that encourage people with chronic low back pain (pain $>3$ months) to assume a physically active role in their recovery [3-6]. However, these patients often report decreased habitual physical activity levels, believing that if movement hurts they may be re-injuring themselves, termed 'fear avoidance' $[7,8]$.

The recent European Clinical Guidelines for CLBP concluded that supervised group exercise is an attractive firstline option for treating large numbers of CLBP patients at low cost [6]. The "Back to Fitness" physiotherapy-led supervised group exercise programme for CLBP was introduced in the UK in the 1990s [9]. Its effectiveness has been supported in several RCTs, reporting significant improvements in pain and disability compared to 'routine' physiotherapy (i.e. advice/education, passive mobilisation/manipulation)[10] and GP management [11], and it has been shown to be cost effective[11]. Nonetheless, a national survey by the Principal Investigator of public general hospitals in the Republic of Ireland (ROI), found that only $39 \%$ of responding physiotherapy departments were delivering group-based exercise programmes for CLBP, the main barriers being space and time restrictions, and insufficient staffing levels [12].

Furthermore, another limiting factor from the patients' perspective is poor adherence with the recommended exercises [13], and the requirement for regular attendance at the class with drop out rates of up to $30 \%$ being reported in the literature [14]. Given the difficulties and limited availability of supervised exercise programmes, an alternative clinically and cost effective approach to increasing the activity levels of patients with CLBP is warranted.

The European Clinical Guidelines have identified the use of brief/minimal contact self-activation interventions that encourage participation in physical activity for CLBP as an area for future research, particularly as this approach could result in significant cost savings if it proves to be at least as effective as other treatments [6]. For CLBP, there is moderate evidence from RCTs $[15,16]$ and a systematic review [3] that brief information and advice to stay active are more effective than usual GP care in reducing LBPrelated disability, but not pain levels. However, there is limited evidence of the effects of self-activation interventions compared to supervised exercise programmes on pain and disability levels [17], and no evidence of the effects of either type of programme in increasing CLBP patients' level of participation in physical activities, return to work rates or psychosocial variables compared to 'routine' physiotherapy.

Walking may be an ideally suited form of exercise prescription as it is easy to do, requires no special skills or facilities, and is achievable by virtually all ages with little risk of injury $[18,19]$. General physical activity recommendations encourage individuals to accumulate $30 \mathrm{~min}$ utes of moderate intensity physical activity on five days per week [20] or 10,000 steps [21]; and there is evidence from a meta-analysis that healthy but sedentary individuals who take up a programme of regular brisk walking improve several known risk factors for cardiovascular disease [22]. There is limited evidence that people with LBP are less physically active and have altered patterns of physical activity than matched controls $[23,24]$. Several recent studies have reported that people with chronic LBP failed to reach the recommended 10,000 steps per day and took significantly less steps than healthy age-matched controls $[24,25]$.

There has been minimal investigation of the effectiveness of walking programmes in LBP management. One RCT found unsupervised walking at a self-selected pace was less effective than individual physiotherapy or medical exercise therapy in reducing pain, disability, costs and increasing patient satisfaction levels in people with CLBP [26]. Participants in the walking group were instructed to walk for one hour three times per week at their convenience, preferably on alternate days. However, apart from one information session, participants in the walking group received no further contact or support from a health 
professional, and their level of compliance or physical activity was not investigated. From a North American study back pain sufferers who participated in brisk walking for at least 3 hours per week reported reductions in low back pain, disability, and psychological distress [27]. Nonetheless, that trial was primarily designed to compare chiropractic and medical care for LBP, and thus patients did not receive any structured physical activity or exercise interventions and no details of the walking intervention are provided. Given the limited research to support or refute walking programmes in CLBP management, there is a need to investigate the effectiveness of such programmes in people with chronic LBP.

For the researchers designing such walking programmes, and the clinicians delivering them, the challenge is to devise an effective intervention that will motivate habitually sedentary people with LBP to be physically active. A Cochrane systematic review of interventions to promote physical activity concluded that a mixture of self-direction and on-going professional support can encourage adults to be more physically active [28]. A randomised controlled trial found that an eight-week low frequency progressive walking programme for 'normal' sedentary adults had high levels of adherence, and resulted in increased physical activity levels with no adverse effects or injuries, thus increasing its clinical application [29]. Another trial reported evidence for the benefit to fitness and cardiovascular risk of the ' 30 minute brisk walking, five days per week' message to people aged 50-65 years who participated in a 12 week unsupervised home-based walking programme that used pedometers as motivational tools [30]. Pedometers are simple to use, inexpensive devices that produce a user-friendly output (step count, duration) that serves as an effective motivator to increase physical activity [31].

A self-activation programme by walking may be a more pragmatic and less costly intervention than supervised exercise classes to promote increased physical activity levels in patients with CLBP. It has the additional advantages of supporting a larger population of CLBP patients at the same time, and by moving the emphasis away from the hospital setting could reduce the costs to the Health Service. Given that the majority of patients with CLBP currently receive a range of interventions within the scope of 'usual' physiotherapy (advice, passive mobilization/ manipulation, general exercise [32-34], this RCT will establish the difference in effectiveness of a walking programme and a supervised general exercise programme compared to usual physiotherapy, which will act as the control intervention, in subjects with chronic low back pain.

The primary objective is to: (i) determine the difference between groups in mean change in functional disability at 6 months

The secondary objectives are to:

(ii) determine the difference between groups in mean changes in pain, health-related quality of life, psychosocial beliefs, days of sick leave, daily physical activity levels, self efficacy, readiness to change and patient satisfaction

(iii) determine the difference between groups in cost utility and the cost effectiveness of the alternative treatment programmes

(iv) determine the difference between groups in level of adherence to each intervention

(v) complete a qualitative exploration of subjects experience of each intervention

\section{Hypotheses Tested}

The trial will test two 'null' hypotheses. The primary null hypotheses is that,

(a). in people referred for physiotherapy for chronic LBP there is no difference in clinical outcome and costs between those receiving a walking programme, a supervised general exercise programme and usual physiotherapy;

The secondary null hypothesis is that:

(b). subjects that have received a walking programme or supervised general exercise programme will not have changed daily physical activity levels compared to those who have received usual physiotherapy

\section{Methods/design}

The Research Ethics Committees of the participating Dublin hospitals have granted approval for this study:

1. Adelaide and Meath Hospital incorporating the National Children's Hospital

2. Beaumont Hospital

3. Connolly Hospital

4. Mater Misericordiae University Hospital

5. St Vincent's University Hospital

The trial will be reported according to the recommendations of the CONSORT statement [35] and the flow of par- 
ticipants through the study is represented in Figure 1. The quantitative study will establish the difference in clinical effectiveness and costs of a walking programme and a supervised general exercise programme compared to usual physiotherapy, which will act as the control intervention, for patients with chronic low back pain. The qualitative study will explore participants' experience of the study and the interventions.

\section{Quantitative study}

\section{Design}

The study will be a prospective randomized controlled trial (RCT) with three arms (i) walking programme, (ii) a supervised exercise class, (iii) usual physiotherapy of up to 8 weeks duration. Outcomes will be assessed at base- line and 3, 6 and 12 months [follow-up by pre-paid postage].

\section{Controlling bias}

The RCT design includes key methodological features that have been recognized as important in minimizing bias in clinical trials: true randomization, concealed allocation, specification of eligibility criteria, blind outcome assessment, blind analysis and intention-to-treat analysis.

\section{Setting}

A sample of patients will be recruited in Dublin, Ireland through the physiotherapy departments of acute public hospitals that provide physiotherapy treatment for people with CLBP.

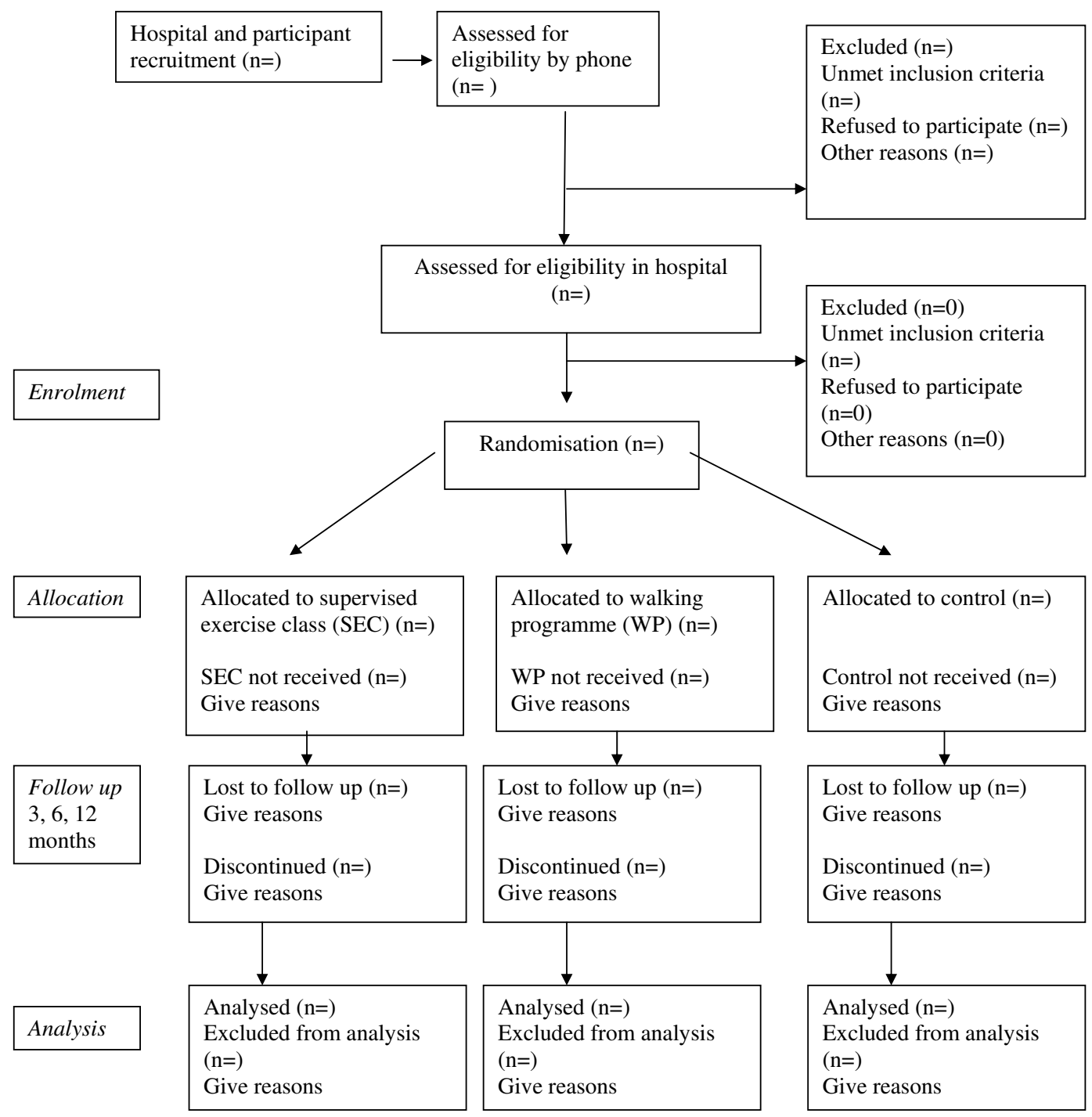

\section{Figure I}

Participant flow through the RCT (based on CONSORT statement). 


\section{Protocol protection}

The following mechanisms will be used to ensure the trial protocol is applied consistently: protocol manuals will be developed and all involved researchers and clinicians will be trained to ensure that subject screening, assessment, random allocation and treatment procedures are conducted according to the protocol; a random sample of $20 \%$ of treatment records in each group will be audited by a researcher not involved in the day to day running of the trial to check that treatment is administered as per the protocol; if any anomalies are found all treatment records will be checked. The treatment record forms will be completed by clinicians on every patient recruited to the study.

\section{Study population and recruitment Clinics and Clinicians}

The study will be conducted in several Dublin metropolitan acute general hospitals physiotherapy departments that provide rehabilitation for musculoskeletal conditions. The relevant hospitals will be contacted by telephone and email seeking expressions of interest to participate in the study. A meeting will be scheduled with each hospital's physiotherapy department manager to outline the project and distribute the outline protocol. Information meetings will be arranged with managers and treating physiotherapists to discuss the study background, aims and methodologies and to address their queries. Reception staff will be briefed on the study recruitment process, and patient appointment systems, paperwork and filing arrangements will be made. All participating physiotherapists involved in the interventions will attend training days in the School of Physiotherapy and Performance Science, University College Dublin. These days will be delivered by various members of the research team. The main focus of the training days will be to ensure that the interventions are standardized across all centres. A cognitive behavioural therapy (CBT) approach will be emphasized and performance indicators will be set for the physiotherapists to further ensure homogeneity of the active interventions.

In advance of the training days, a detailed trial manual will be distributed to the therapists. Their role in the study will be highlighted. They will also complete the PABs PT (Pain Attitudes and Beliefs of Physiotherapists) Questionnaire to establish their biopsycho-social or biomedical orientation to the management of CLBP [36].

\section{Patients}

All eligible patients with chronic or recurrent LBP referred to the participating hospitals physiotherapy departments by general practitioners or hospital consultants will be invited to participate in the study by the Trial Co-ordinator in order of referral until adequate subject numbers are achieved. Patients will receive the initial contact by tele- phone to explain the trial procedures, to clarify interest and to screen eligibility, and will then be followed up by a letter containing the patient information sheet and an invitation to attend for baseline assessment. Suitable, interested patients will attend the relevant hospital's physiotherapy department, where detailed verbal explanations of the study protocol will be provided and, written informed consent will be sought by the Trial Co-ordinator. The Trial Co-ordinator will record the number of patients invited, the number who declined, ineligible patients and reasons.

\section{Eligibility Assessment Clinicians}

Chartered Physiotherapists who are eligible for membership of the Irish Society of Chartered Physiotherapists and are employed by one of the participating hospitals are eligible to participate.

\section{Patients}

At the initial telephone contact and the baseline interview the Trial Co-ordinator will use a screening checklist to verify eligibility (Table 1). The Physical Activity Readiness Questionnaire (PAR-Q)[37] will be completed to determine whether medical clearance is necessary before trial participation.

\section{Randomisation}

Patients who have consented to participate will be randomly allocated in accordance with recognised procedures, by computer-generated random allocation sequences that will be prepared centrally by the trial statistician. This sequence will be used to randomly allocate each consenting, numbered subject to one of three study groups: supervised exercise class, walking programme or usual physiotherapy.

Stratification will be by hospital only and separate randomisation lists will be used for each hospital. Prior to randomisation each subject's group allocation preference will be sought and recorded by the Trial Co-ordinator in order to investigate whether treatment preference has any influence on outcomes.

The Trial Co-ordinator will telephone/text the Research Administrator who will provide a central, remote, telephone randomisation service, to obtain each consecutive subject's random group allocation. Following the baseline assessment an appointment for the relevant intervention will then be made by the Trial Co-ordinator. Each participant will receive a copy of 'The Back Book'[38] and be advised to read it before the first physiotherapy appointment. Participants allocated to the WP will be given an educational walking manual, and a Yamax Digiwalker Pedometer, instructed in its use and requested to wear it 
Table I: Eligibility Criteria for the SWIFT Trial

\begin{tabular}{|c|c|}
\hline Inclusion criteria & Exclusion criteria \\
\hline $\begin{array}{l}\text { Patients with chronic ( } \geq 3 \text { months) or recurrent ( } \geq 3 \text { episodes in previous } \\
12 \text { months) LBP of mechanical origin with/without radiation to the } \\
\text { lower limb }\end{array}$ & $\begin{array}{l}\text { Currently or having received treatment for CLBP within previous } 3 \\
\text { months }\end{array}$ \\
\hline Males/females between 18-65 years & $\begin{array}{l}\text { Red flags indicating serious spinal pathology, e.g. cancer, cauda equina } \\
\text { lesion }\end{array}$ \\
\hline No spinal surgery within the previous 12 months & Radicular pain indicative of nerve root compression \\
\hline $\begin{array}{l}\text { Patients deemed suitable by their GP/hospital consultant to carry out an } \\
\text { exercise programme }\end{array}$ & $\begin{array}{l}\text { Patients diagnosed with severe spinal stenosis, spondylolisthesis, } \\
\text { fibromyalgia }\end{array}$ \\
\hline $\begin{array}{l}\text { Patients willing to attend for an 8-week treatment programme of } \\
\text { exercise classes }\end{array}$ & History of systemic/inflammatory disease, e.g. rheumatoid arthritis \\
\hline Access to a telephone (for follow-up support) & $\begin{array}{l}\text { Patients with any confounding conditions such as a neurological disorder } \\
\text { or currently receiving treatment for cancer }\end{array}$ \\
\hline Fluency in English (verbal and written) & $\begin{array}{l}\text { Patients with acute }(<6 \text { weeks) or subacute LBP }(6-12 \text { weeks), provided } \\
\text { that they have experienced }<3 \text { LBP episodes during previous } 12 \text { months }\end{array}$ \\
\hline \multirow[t]{3}{*}{$\begin{array}{l}\text { 'Low' or 'moderate' levels of physical activity measured by the IPAQ } \\
\text { (<600 MET-minutes/week) }\end{array}$} & $\begin{array}{l}\text { Unstable angina/uncontrolled cardiac dysrhythmias/severe aortic } \\
\text { stenosis/acute systemic infection accompanied by fever }\end{array}$ \\
\hline & Medico-legal issues \\
\hline & Pregnancy \\
\hline
\end{tabular}

for seven days to record number of steps and habitual daily activity levels (frequency of walks, walk duration) in an exercise diary prior to the start of the intervention.

\section{Blinding}

\section{Clinicians}

Due to the nature of the interventions it will not be possible to blind the participating clinicians to the group allocation of patients, but clinicians will not be involved in outcome assessment.

\section{Patients}

Due to the nature of the interventions it will not be possible to blind patients to their group allocation. The patient information leaflet will inform participants that they have an equal chance of receiving one of three physiotherapy approaches for low back pain management.

\section{Assessors}

A blinded researcher will administer all outcome measures for postal follow-up. The Trial Co-ordinator will not be involved in follow-up outcome assessment. The statistician will be unaware of group allocation until completion of data analyses.

\section{Sample Size}

A total of 189 patients ( $\mathrm{n}=63$ per group) will be required for $80 \%$ power to detect a 4 point difference between groups on the primary outcome, the Oswestry Disability Index [39], with 95\% confidence using a two-tail between-within repeated measures ANOVA test of difference between group means. Based on the results of previous LBP trials investigating exercise [11] and using postal follow-ups [40], we anticipate a $30 \%$ loss to follow-up. Thus we aim to recruit 82 subjects per group (total 246 subjects).

\section{Outcome Measures}

At the baseline assessment sociodemographic data (i.e. age, gender, education level, social status, occupation and work status, past medical history, LBP history), cardiorespiratory fitness using the shuttle walk test [41], blood pressure, Body Mass Index (BMI, kg/ $\mathrm{m}^{2}$ ) and any previous treatment will be documented by the Trial Coordinator. A combination of recommended self-report valid and reliable outcome questionnaires [42] detailed below, and objective measurement will be used at baseline and follow-up (3, 6 and 12 months). The satisfaction questionnaire will only be administered at the end of scheduled intervention period (3 months). 


\section{Clinical Outcomes}

The primary outcome measure will be a change in:

(i) Functional disability due to LBP measured by the Oswestry Disability Index (ODI) [43]

The secondary outcome measures will be changes in:

(ii) Pain using Numerical Rating Scales for current and worst pain [44]

(iii) Health-related quality of life measured by the EuroQol questionnaire [45]

(iv) Psycho-social beliefs using the Fear Avoidance Beliefs Questionnaire [46] and the Back Beliefs Questionnaire [47]

(v) Employment status and number of days reported sick leave over the past year for those in paid employment only

(vi) Self-report physical activity levels using the International Physical Activity Questionnaire (IPAQ) [48],

(vii) Objective physical activity levels using an activPal ${ }^{\mathrm{TM}}$ device: a valid and reliable measure of walking that is capable of recording steps, time spent lying/sitting, standing and stepping under free living conditions [49] previously used by some of the authors in another LBP trial [50]. A sample of convenience of participants from each group will be requested to wear the activPAL ${ }^{\mathrm{TM}}$ on 3 separate occasions each lasting 7 days (1) at baseline, (2) 3 months and (3) 6 months after initial randomisation.

(viii) Self efficacy questionnaire [51]

(ix) Readiness to change questionnaire [52]

(x) Patient satisfaction will be assessed using Likert scales assessing satisfaction with outcome and satisfaction with care at 3 months [53]

\section{Cost Outcomes}

Two cost outcomes will be used

(i) Participants resource utilization will be assessed using cost diaries developed and successfully used by the Principal Investigator in a previous RCT [54]. A random sample of $20 \%$ of cost diaries in each group will be verified by telephone interview with a researcher not involved in the day to day running of the trial to check the accuracy and ensure the quality of the data. (ii) Participant's health state will be assessed using the EuroQol (EQ-5D) Questionnaire's Weighted Health Index [45] and Visual Analogue Scale.

Follow-up reminders will be given by phone and completed questionnaires returned by pre-paid envelopes.

\section{Interventions}

(i) Supervised exercise class (SEC)

Within one week of randomisation, participants will commence the SEC. This class will follow a group-based format based on the 'Back to Fitness' programme used in the UK BEAM trial [9-11,13,14] which is underpinned by cognitive behavioural therapy principles designed to change participants behaviour by modifying their attitude to their LBP, i.e. 'hurt' does not mean harm $[7,8]$. First, each participant will attend the physiotherapy department for an initial individual assessment with the Chartered Physiotherapist delivering the class, where there will be discussion and agreement between the therapist and the patient on short and long-term goals; recording of the patient's exercise capabilities and perceived barriers to recovery and the individual's treatment expectations. Second, participants will attend the physiotherapy department of the relevant participating hospital once a week for 8 weeks for a one-hour supervised group exercise class led by a Chartered Physiotherapist. The physiotherapist will advise patients according to their individual goals and exercise capabilities, and help identify which exercise(s) they could continue independently of the treatment sessions, i.e. foster the development of self-management strategies. Subjects will also be required to rate their perceived exertion during the class on the Borg scale - a linear scale measuring level of breathlessness from $0=$ 'not breathless at all' to $10=$ 'maximal'. [55,56]. Patients will be encouraged to accept responsibility for determining and carrying out their weekly programme of activity. Adherence with the supervised exercise programme will be recorded as the number of sessions attended. The number of sessions defined as adherence will be decided on completion of the trial.

\section{(ii) Walking programme (WP)}

Within one week of randomisation, participants will commence the WP, the focus being to increase physical activity through a graded walking programme. The WP is based on previous effective programmes in healthy sedentary adults $[29,30,57,58]$ as no previous programmes for LBP are available.

As with the SEC, each participant will attend the physiotherapy department for an initial individual assessment, where there will be discussion and agreement between the therapist and the patient on short and long-term goals; recording of the patient's exercise capabilities and per- 
ceived barriers to recovery and the individual's treatment expectations. The therapist will use the information recorded in the exercise diary to inform the starting point for the eight week progressive WP; the minimum being a 10 minute walk (approx 1200 steps) on at least four days per week to be decided with, where possible, one day's rest between walks. The aim of the programme is to progress to the American College of Sports Medicine guidelines of 30 minutes moderate intensity walking on five days per week by week five [20], and then to maintain this level for the remainder of the programme. The 30 minutes brisk walking may be accumulated in two or three shorter bouts if this is more attainable e.g. three 10 minute walks $[30,58]$. A recent review found no difference in the positive effects on cardiovascular fitness of empirical studies of accumulated or continuous physical activity in sedentary adults and highlighted the need for research to evaluate if accumulated exercise may increase compliance in previously sedentary adults [59]. All participants will be encouraged to use the Borg Breathlessness scale to establish their walking speed: targeting level three (moderate breathlessness) to four (somewhat severe), the minimum level required to achieve the benefits related to exercise $[55,56]$.

Participants will be contacted once per week by telephone by the Chartered Physiotherapist who performed the initial assessment to progress their walking frequency and duration based on their exercise diary record of the previous week's walking, and to provide encouragement. These telephone calls will be based around a specifically developed telephone script based upon CBT principles. Participants will be advised that just like athletes, any unaccustomed exercise is likely to produce some muscle soreness [8]. Participants will use their Yamax Digiwalker Pedometer [60] as a motivational feedback tool, providing immediate information on activity levels [61].

Adherence with the walking programme will be assessed by the frequency, distance, number of steps taken and duration of walks recorded in the exercise diary. Specific adherence levels will be established once the trial is complete. At the end of the intervention subjects will reattend the physiotherapy department for a review appointment with a view to discharge from physiotherapy.

\section{(iii) Usual Physiotherapy (UP) -Control Group}

Within one week of randomization, participants will commence individual usual physiotherapy at the discretion of the treating physiotherapist in the participating hospital. All physiotherapy treatments and the number of visits will be recorded for the study period in previously designed treatment record forms. On the basis of a previous RCT by the Principal Investigator in the Republic of Ireland public physiotherapy health service the anticipated mean (SD) number of treatments is 5.8 over a mean (SD) of 7.7 weeks (5.8) weeks [54]. A multimodal approach of education/advice, manipulative therapy and exercise therapy will be permitted on the basis of the results of previous surveys of physiotherapy practice in the UK and Ireland [32-34]. As part of this it is expected that subjects will be provided with an individualized exercise programme at the discretion of the treating therapist but will not be permitted to attend group exercise class or undertake a walking programme during the trial. Adherence will be assessed by the number of visits prior to discharge from physiotherapy.

\section{Adverse effects or events}

No adverse events, apart from minor musculoskeletal complaints in the WP group, are anticipated but will be documented by type, length of time, and frequency should they occur [62].

\section{Data Analysis}

Analysis will be by intention to treat. All participants and group allocation will be coded, sociodemographic data coded, and outcome questionnaires scored, and all data entered into the Statistical Package for the Social Sciences database for analysis following data cleaning and checking for errors. Analysis of the clinical outcome data will be performed by the statistician who will remain blinded to group identification until analysis is complete. Between group analyses will be performed to test for between group differences in each outcome from baseline at follow-up. Continuous outcome variables will be analysed using a mixed between-within repeated measures analysis of variance if the assumptions for parametric statistics hold. If substantial evidence of non-normality is found appropriate alternative (non-parametric) tests will be applied, and specified as the primary analysis method.

Using data from the cost diaries, the extent to which each of the interventions has impacted on subsequent use of health care will also be calculated and will cover all identifiable health care costs. The direct and indirect costs to patients receiving each intervention will be obtained. The health care services costs incurred during the intervention and year to follow up will be calculated using the relevant observable prices. Where prices cannot be observed shadow prices will estimated; mean and median health care utilization rates and associated direct service costs in addition to indirect and direct patient costs will be calculated for both intervention groups. Relationships between presenting LBP severity and utilization/cost will be investigated. An additional cost effectiveness analysis will use the magnitude of a mean and median change on the Oswestry Disability Questionnaire as the natural unit of outcome.

The resulting scores from the EQ-5D health state and the visual analog scale will be used to generate the health state 
utility values necessary for the within trial cost utility analysis (CUA). The CUA will allow the outcomes of this study to be compared to other studies allowing the relevant short term benefit of the interventions to be assessed.

\section{Qualitative study}

A sample of participants from each group will be invited to participate in a semistructured telephone interview or to attend a focus group (one per intervention) at the end of the 6 month follow-up. These subjects will be contacted by letter and invited to contact the Trial Co-ordinator if they wish to participate. Both the focus group and telephone interviews will be conducted by an experienced interviewer with a predetermined set of questions. A "clue and process" format using a checklist of topics, will be used to ensure that the same basic areas are covered but allowing any issues of importance to the participants to emerge. The sessions will be audiotaped, minuted and transcribed verbatim for independent analysis of emergent themes. The main areas to be explored will be subjects' reasons for participation in the trial, their interpretation of study information and documentation, their experiences, expectations and satisfaction with the programme of care including barriers/motivators to participation in the relevant programme.

\section{Data Analysis}

Qualitiative data from the telephone interviews and focus groups will be analysed using Burnard's thematic analysis [63]. Emerging themes will be identified and comparisons explored between patients' experience of trial participation, their perception of treatment effectiveness and response to each intervention, its impact, motivators and barriers to adherence, as well as their expectations and treatment preferences. A random sample of transcripts from each group will be selected and reviewed by an independent researcher not otherwise involved in the study for inter-rater and intra-rater reliability of identified themes.

\section{Discussion}

We have presented the rationale and design of a randomised controlled trial, with embedded qualitative and economic studies, to investigate and evaluate the difference in effectiveness of a walking programme and a supervised general exercise programme compared to usual physiotherapy, which will act as the control intervention, in subjects with chronic low back pain. The results of this study will be presented as soon as they are available.

\section{Abbreviations}

The following abbreviations have been used in the manuscript: LBP: low back pain; CLBP: chronic low back pain; RCT: randomized controlled trial; CBT: cognitive behavioural therapy; UK: United Kingdom; SEC: supervised exercise class; WP: walking programme.

\section{Competing interests}

The authors declare that they have no competing interests.

\section{Authors' contributions}

All authors were involved in the design of the study. DHO will act as Principal Investigator and was responsible for drafting the paper, and all authors commented on the draft. All authors have read and approved the final manuscript.

\section{Acknowledgements}

The Health Research Board Project Grant 2007/79 funded this research. The authors wish to thank all hospitals, clinicians and patients for their participation in this project and Mr Rónán Doherty, Ms Clare Kelly and Ms Ruth Mills for data collection. The authors wish to acknowledge Mr Aodán Tynan for advice about cost analysis, and $\mathrm{Dr}$ Chris Lonsdale and $\mathrm{Dr}$ Suzanne Guerin for advice about qualitative study data analysis.

\section{References}

I. Croft PR, Dunn KM, Raspe H: Course and prognosis of back pain in primary care: the epidemiological perspective. Pain 2006, I 22(I-2): 103.

2. Waddell G, Aylward M: The scientific and conceptual basis of incapacity benefits London TSO; 2005.

3. Hayden JA, van Tulder MW, Tomlinson G: Systematic review: strategies to improve outcomes in chronic low back pain. Ann Intern Med 2005, I42(9):776-785.

4. van Tulder MW, Hayden JA, van Tulder MW, Malmivaara A, Koes BW: Exercise therapy for treatment of non-specific low back pain. Cochrane Database Syst Rev 2005:CD000335. Review

5. Chou R, Qaseem A, Snow V, Casey D, Cross JT Jr, Shekelle P, Owens DK: Clinical Efficacy Assessment Subcommittee of the American College of Physicians; American College of Physicians; American Pain Society Low Back Pain Clinical Guidelines Panel: Diagnosis and treatment of low back pain: a joint clinical practice guideline from the American College of Physicians and the American Pain Society. Ann Intern Med 2007, |47(7):478-9|.

6. Airaksinen O, Brox Jl, Cedraschi C, Hildebrandt J, Klaber-Moffett J, Kovacs F, Mannion AF, Reis S, Staal JB, Ursin H, Zanoli G: COST B I3 Working Group on Guidelines for Chronic Low Back Pain: Chapter 4. European guidelines for the management of chronic nonspecific low back pain. Eur Spine J 2006, I5(Suppl 2):S192-300.

7. Klaber-Moffett J, Carr J, Howarth E: High fear-avoiders of physical activity benefit from an exercise program for patients with back pain. Spine 2004, 29: I I67-I I72.

8. Pincus T, Vogel S, Burton AK, Santos R, Field AP: Fear avoidance and prognosis in back pain: a systematic review and synthesis of current evidence. Arthritis Rheum 2006, 54(I 2):3999-40I0.

9. Klaber-Moffett J, Frost H: Back to Fitness: The manual for physiotherapists to set up classes. Physiotherapy 2000, 86(6):295-305.

10. Frost H, Klaber-Moffett JA, Moser JS, Fairbank JCT: Randomised controlled trial for evaluation of fitness programme for patients with chronic low back pain. BMJ 1995, 3 1 0:15I-I54.

II. Klaber-Moffett J, Torgensen D, Bell-Syer S, Jackson D, Llewlyn-Phillips $H$, Farrin A, Barber J: Randomised controlled trial of exercise for low back pain: clinical outcomes, costs and preferences. BMJ 1999, 319:279-283.

12. Byrne K, Doody C, Hurley DA: Exercise therapy for low back pain: A small-scale exploratory survey of current physiotherapy practice in the Republic of Ireland acute hospital setting. Man Ther 2006, I I(4):272-278.

13. Carr JL, Klaber-Moffett JA, Howarth E, Richmond SJ, Torgensen DJ, Jackson DA, Metcalfe CJ: A randomised trial comparing a group exercise programme for back pain patients with individual physiotherapy in a severely deprived area. Disab Rehab 2005, 27(16):929-937.

14. UK BEAM Trial Team: United Kingdom back pain exercise and manipulation (UK BEAM) randomised trial: effectiveness of 
physical treatments for back pain in primary care. BMJ 2004, I I;329(7479): I 377 .

15. Indahl A, Haldorsen EH, Holm S, Reikeras O, Ursin H: Five year follow up study of a controlled trial using light mobilisation and an informative approach to low back pain. Spine 1998, 23(23):2625-30

16. Frost H, Lamb SE, Doll H, Rivero-Arias O, Taffe Carver PT, StewartBrown S: Randomised controlled trial of physiotherapy treatments versus advice for low back pain: Clinical effectiveness and cost. BMJ 2004, 329(7468):708. Epub 2004 Sep 17

17. Storheim K, Brox Jl, Holm I, Koller AK, Bo K: Intensive group training versus cognitive intervention in subacute low back pain: short term results of a single blind randomised controlled trial. J Rehabil Med 2003, 35(3): $132-140$.

18. National Institute of Clinical Excellence: Four commonly used methods to increase physical activity. London: NICE; 2006.

19. Siegel PZ, Brackbill RM, Heath GW: The epidemiology of walking for exercise: implications for promoting activity among sedentary groups. Am J Pub Health 1995, 85:706- 10.

20. American College of Sports Medicine: ACSM's Guidelines for Exercise Testing and Prescription Philadelphia: Lippincott Williams and Wilkins; 2006.

21. Tudor-Locke C, Hatano Y, Pangrazi RP, Kang M: Revisiting "how many steps are enough?". Med Sci Sports Exerc 2008, 40(7 Suppl):s537-43

22. Murphy MH, Nevill AM, Murtagh EM, Holder RL: The effect of walking on fitness, fatness and resting blood pressure: a meta-analysis of randomised, controlled trials. Prev Med 2007, 44(5):377-85.

23. Verbunt JA, Westerterp KR, Heijden GJ van der, Seelen HA, Vlaeyen JW, Knottnerus JA: Physical activity in daily life in patients with chronic low back pain. Arch Phys Med Rehab 200I, 82:726-730.

24. Ryan CG, Grant PM, Dall PM, Gray H, Newton M, Granat M: Individuals with chronic low back pain have a lower level, and an altered pattern of physical activity compared with matched controls: an observational study. Aust J Physiother 2009, 55(I):53-58

25. Hurley DA, Brady L, O'Brien E, McDonough SM, Baxter GD, Heneghan C: Subjective and objective evaluation of the physical activity profiles of people with low back pain and agematched controls over 7-days. J Bone Joint Surg Br Proceedings in press.

26. Torstensen TA, Ljunggren AE, Meen HD, Odland E, Mowinckel P, Geijerstam S: Efficiency and costs of medical exercise therapy, conventional physiotherapy and self exercise in patients with chronic low back pain. Spine 1998, 23:2616-2624.

27. Hurwitz EL, Morgenstren H, Kominski GF, Yu F, Chiang LM: A randomised trial of chiropractic and medical care for patients with low back pain: eighteen months follow up outcomes from the UCLA low back pain study. Spine 2006 , $3 I(6): 6||-62 \mid$.

28. Hillsdon M, Foster C, Thorogood M: Interventions for promoting physical activity. Cochrane Database Syst Rev 2005, 25(I):CD003I80.

29. Murphy MH, Murtagh EM, Boreham CA, Hare LG, Nevill AM: The effect of a worksite based walking programme on cardiovascular risk in previously sedentary civil servants. BMC Public Health 2006, 6:136.

30. Tully MA, Cupples ME, Chan WS, McGlade K, Young IS: Brisk walk ing, fitness, and cardiovascular risk: a randomized controlled trial in primary care. Prev Med 2005, 41 (2):622-8.

31. Bravata DM, Smith-Spangler C, Sundaram V, Gienger AL, Lin N, Lewis $R$, Stave $C D$, Olkin I, Sirard JR: Using pedometers to increase physical activity and improve health: A systematic review. JAMA 2007, 298(19):2296-304.

32. Casserley-Feeney SN, Bury G, Daly L, Hurley DA: Physiotherapy for low back pain: Differences between public and private healthcare sectors in Ireland. Man Ther 2008, I3(5):44 I-9.

33. Foster NE, Thompson KA, Baxter GD, Allen JM: Management of non-specific low back pain by physiotherapists in Britain and Ireland. Spine 1999, 24(13): 1332-1342.

34. Gracey JH, McDonough SM, Baxter GD: Physiotherapy management of low back pain: A survey of current practice in Northern Ireland. Spine 2002, 27(4):406-4II

35. Zwarenstein M, Treweek S, Gagnier J], Altman DG, Tunis S, Haynes $B$, Oxman AD, Moher D, CONSORT group; Pragmatic Trials in
Healthcare (Practihc) group: Improving the reporting of pragmatic trials: an extension of the CONSORT statement. $B M]$ 2008, 337:a2390.

36. Houben RM, Ostelo RW, Vlaeyen JW, Wolters PM, Peters M, Stompvan den Berg SG: Health care providers' orientations towards common low back pain predict perceived harmfulness of physical activities and recommendations regarding return to normal activity. Eur J Pain 2005, 9(2): 173-83.

37. Jamnik VK, Gledhill N, Shephard RJ: Revised clearance for participation in physical activity: greater screening responsibility for qualified university-educated fitness professionals. Appl Physiol Nutr Metab 2007, 32(6): I191-7.

38. Back Book London: HMSO Stationery Office; 1996.

39. Frost H, Lamb SE, Stewart-Brown S: Responsiveness of a patient specific outcome measure compared with the Oswestry Disability Index v2.I and Roland and Morris Disability Questionnaire for patients with subacute and chronic low back pain. Spine 2008, 33(22):2450-7.

40. Hurley DA, McDonough SM, Dempster M, Moore AP, Baxter GD: A randomized clinical trial of manipulative therapy and interferential therapy for acute low back pain. Spine 2004, 29(20):2207-16.

4I. Taylor S, Frost H, Taylor A, Barker K: Reliability and responsiveness of the shuttle walking test in patients with chronic low back pain. Physiother Res Int 200I, 6(3): I70-178.

42. Bombardier C: Outcome assessments in the evaluation of treatment of spinal disorders: summary and general recommendations. Spine 2000, 24(25):3100-3103.

43. Roland M, Fairbank J: The Roland-Morris Disability Questionnaire and the Oswestry Disability Questionnaire. Spine 2000, 25(24):3। I5-24.

44. Melzack R, Katz J: Pain assessment in adult patients. In Wall and Melzack's Textbook of Pain 5th edition. Edited by: McMahon SB, Koltzenburg M. Amsterdam: Elsevier; 2006.

45. EuroQol Group: EuroQol: a new facility for the measurement of health related quality of life. Health Policy 1990 , 16(3): 199-208.

46. Waddell G, Newton M, Henderson I, Somerville D, Main CJ: A FearAvoidance Beliefs Questionnaire (FABO) and the role of fear-avoidance beliefs in chronic low back pain and disability. Pain 1993, 52:157-168.

47. Symonds TL, Burton AK, Tillotson KM, Main CJ: Do attitudes and beliefs influence work loss due to low back trouble? Occup Med (Lond) 1996, 46(I):25-32.

48. Craig CL, Marshall AL, Sjöström M, Bauman AE, Booth ML, Ainsworth BE, Pratt M, Ekelund U, Yngve A, Sallis JF, Oja P: International physical activity questionnaire: I 2-country reliability and validity. Med Sci Sports Exerc 2003, 35(8): 138I-95.

49. Ryan CG, Grant PM, Tigbe WW, Granat MH: The validity and reliability of a novel activity monitor as a measure of walking. $\mathrm{Br}$ J Sports Med 2006, 40(9):779-784.

50. McDonough SM, Liddle SD, Hunter R, Walsh DM, Glasgow P, Gormley G, Hurley D, Delitto A, Park J, Bradbury I, Baxter GD: Exercise and manual auricular acupuncture: a pilot assessor-blind randomised controlled trial. (The acupuncture and personalised exercise programme (APEP) trial). BMC Musculoskelet Disord 2008, 9:31.

51. Schwarzer R, Jerusalem M: Generalised Self-Efficacy scale. In Measures in Health Psychology Portfolio Edited by: Wright S, Johnston M, Weinman J. Windsor: NFER-Nelson; 1995.

52. Marcus BH, Rossi JS, Selby VC, Niaura RS, Abrams DB: The stages and processes of exercise adoption and maintenance in a worksite sample. Health Psychol I992, I I (6):386-95.

53. Hills R, Kitchen S: Satisfaction with outpatient physiotherapy. A survey comparing the views of patients with acute and chronic musculoskeletal conditions. Physiother Theory Pract 2007, 23:2I-36.

54. Casserley-Feeney SN, Bury G, Daly L, Hurley DA: The Access Trial: Randomised controlled trial of public hospital-based versus private community-based physiotherapy for low back pain: I 2 month follow-up clinical outcome results. J Bone Joint Surg $\mathrm{Br}$ Proceedings in press.

55. Borg GA: Psychophysical bases of perceived exertion. Med $\mathrm{Sc}$ Sports Exerc 1982, 14(5):377-381. 
56. Wilson RC, Jones PW: Long-term reproducibility of Borg scale estimates of breathlessness during exercise. Clin Sci 199I, 80:309-3।2.

57. Tully MA, Cupples ME, Hart ND, McEneny J, McGlade KJ, Chan WS, Young IS: Randomised controlled trial of home-based walking programmes at and below current recommended levels of exercise in sedentary adults. J Epidemiol Community Health 2007, $61(9): 778-83$.

58. Murphy M, Nevill A, Neville C, Biddle S, Hardman A: Accumulating brisk walking for fitness, cardiovascular risk and psychological health. Med Sci Sports Exerc 2002, 34: I468-I 474.

59. Murphy MH, Blair SN, Murtagh EM: Accumulated versus continuous exercise for health benefit: a review of empirical studies. Sports Med 2009, 39(I):29-43.

60. Schneider PL, Crouter SE, Bassett DR: Pedometer measures of free-living physical activity: Comparison of 13 models. Med Sci Sport Exerc 2004, 36(2):33I-335.

61. Tudor-Locke C, Corbin CB, Pangrazi RP, Franks D: Taking Steps Towards Increased Physical Activity: Using Pedometers to Measure and Motivate. President's Council on Physical Fitness and Sports Research Digest 2002, Series 3(I7):

62. Goodrich DE, Larkin AR, Lowery JC, Holleman RG, Richardson CR: Adverse events among high risk participants in a home based walking study: a descriptive study. Int J Behav Nutr Phys Activ 2007, 4:20-32.

63. Burnard P: A method of analysing interview transcripts in qualitative research. Nurse Education Today 1991, I I:46I-466.

\section{Pre-publication history}

The pre-publication history for this paper can be accessed here:

http://www.biomedcentral.com/1471-2474/10/79/pre pub

Publish with Bio Med Central and every scientist can read your work free of charge

"BioMed Central will be the most significant development for disseminating the results of biomedical research in our lifetime. "

Sir Paul Nurse, Cancer Research UK

Your research papers will be:

- available free of charge to the entire biomedical community

- peer reviewed and published immediately upon acceptance

- cited in PubMed and archived on PubMed Central

- yours - you keep the copyright

Submit your manuscript here:

http://www.biomedcentral.com/info/publishing_adv.asp 